Vietnam Journal of Mechanics, VAST, Vol. 29, No. 3 (2007), pp. 375 - 384

Special Issue Dedicated to the Memory of Prof. Nguyen Van Dao

\title{
AN APPROACH TO STUDY VIBRATION IN STOCHASTIC SYSTEMS BASED ON THE ASYMPTOTIC METHOD
}

\author{
NGUYEN Tien KHIEM \\ Institute of Mechanics, VAST
}

\begin{abstract}
The well known Fokker-Plank-Kolmogorov Equation Method has been developed to study random vibration in systems with hysteresis that often described by the stochastic integro-differential equations or differential equations with delay.
\end{abstract}

\section{INTRODUCTION}

The asymptotic method is well-known as one of fundamental methods in study of weakly nonlinear systems. They have come to be effectively used for the stochastic systems via theory of diffusional processes [1]. However, many processes in practice are not diffusional so that for those the Fokker-Plank-Kolmogorov Equation (FPKE) Method is not applicable. In the case, something like the FPKE for non-diffusional processes has been needed. Stratonovich [2] is the first who constructed approximately an equation for probability density function for arbitrary stochastic process based on its asymptotic expansion. It was in fifties of the last millennium. Later, in 1966, Khasminskii [3] had deeper studied the problem in his paper published in Journal of Theory of Probability and Its Application (in Russian). Since 1965, Professor Nguyen Van Dao [4] had published a paper in Vietnamese dealt with an application of the Stratonovich's equation to study random vibration in a weakly nonlinear system. The author of the paper in 1979, after reading Van Dao's work, has came to the idea of developments of the Stratonovich's method to study the processes given by stochastic integro-differential equations. The first result of the author were published in Ukrainian Mathematical Journal in 1983 [5]. This problem were further developed in the author's doctor of science dissertation published in 1991 [8] at the Institute of Mathematics, Ukrainian Academy of Science.

In this paper, some results, taken from the dissertation, are presented to memory of Professor Nguyen Van Dao in the occasion of his $70^{\text {th }}$ celebration.

\section{GENERAL EQUATION FOR PROBABILITY DENSITY FUNCTION OF ARBITRARY STOCHASTIC PROCESS}

Let's consider a $n$-dimensional random process $X=\left\{X_{1}, \ldots, X_{n}\right\}$ with given point $x^{0}=\left\{x_{1}^{0}, \ldots, x_{n}^{0}\right\}$ in the space of states of the process. Characteristic function of the process, as defined, is

$$
\chi\left(u_{1}, \ldots, u_{n}\right)=E\left\{\exp \left[i \sum_{j=1}^{n} u_{j} X_{j}\right]\right\} \equiv\langle\exp [i(u, X)]\rangle .
$$


with the notation $E\{\ldots\} \equiv\langle\ldots\rangle$ implying the probability average operator. Expanding the function $e^{i(u, X)}$ in the Taylor's series at the point $x^{0}$

$$
e^{i(u, X)}=e^{i\left(u, x^{0}\right)}\left\{1+\sum_{j} i u_{j}\left(X_{j}-x_{j}^{0}\right)+\frac{1}{2} \sum_{j, k}\left(i u_{j}\right)\left(i u_{k}\right)\left(X_{j}-x_{j}^{0}\right)\left(X_{k}-x_{k}^{0}\right)+\ldots\right\}
$$

and substituting the obtained expansion to the Eq. (2.1), one will have

$$
\begin{aligned}
\chi(u) \equiv\langle\exp [i(u, X)]\rangle= & e^{i\left(u, x^{0}\right)}\left\{1+\sum_{j} i u_{j}\left\langle X_{j}-x_{j}^{0}\right\rangle\right. \\
& \left.+\frac{1}{2} \sum_{j, k}\left(i u_{j}\right)\left(i u_{k}\right)\left\langle X_{j}-x_{j}^{0}\right\rangle\left\langle X_{k}-x_{k}^{0}\right\rangle+\ldots\right\} .
\end{aligned}
$$

On the other hand, one-point PDF of the process has the form

$$
W(x, t)=(2 \pi)^{-n} \int_{-\infty}^{+\infty} \chi(u) \exp [-i(u, x)] d u_{1} \ldots d u
$$

Substituting the Eq. (2.2) into Eq. (2.3) yields the equation

$$
\begin{aligned}
W(x, t)= & (2 \pi)^{-n} \int_{-\infty}^{+\infty} e^{-i \sum u_{j}\left(x_{j}-x_{j}^{0}\right)}\left\{1+\sum_{j} i u_{j}\left\langle H_{j}\right\rangle\right. \\
& \left.+\frac{1}{2} \sum_{j, k} i u_{j} i u_{k}\left\langle H_{j} H_{k}\right\rangle+\ldots\right\} d u_{1} \ldots d u_{n},
\end{aligned}
$$

where $H_{j}=H_{j}\left(t, x^{0}\right)=X_{j}(t)-x_{j}^{0}, j=1, \ldots, n$. Taking into account the equation

$$
\delta\left(x-x^{0}\right)=(2 \pi)^{-n} \int_{-\infty}^{\infty} \exp \left\{-i \sum_{j} u_{j}\left(x_{j}-x_{j}^{0}\right)\right\} d u_{1} \ldots d u_{n},
$$

Equation (2.4) can be rewritten as

$$
W(x, t)=\left\{\begin{array}{l}
\delta\left(x-x^{0}\right)-\sum_{j} \frac{\partial}{\partial x_{j}}\left\{\left\langle H_{j}\left(t, x^{0}\right)\right\rangle \delta\left(x-x^{0}\right)\right\}+ \\
+\frac{1}{2} \sum_{j, k} \frac{\partial^{2}}{\partial x_{j} \partial x_{k}}\left\{\left\langle H_{j}\left(t, x^{0}\right) H_{k}\left(t, x^{0}\right)\right\rangle \delta\left(x-x^{0}\right)\right\}+\ldots
\end{array}\right\} .
$$

Introducing the operator

$$
L=-\sum_{j} \frac{\partial}{\partial x_{j}}\left\langle H_{j}(t, x)\right\rangle+\frac{1}{2} \sum_{j, k} \frac{\partial^{2}}{\partial x_{j} \partial x_{k}}\left\langle H_{j}\left(t, x^{0}\right) H_{k}\left(t, x^{0}\right)\right\rangle+\ldots
$$

operating as follows

$$
L\{f(x)\}=-\sum_{j} \frac{\partial}{\partial x_{j}}\left\{\left\langle H_{j}(t, x)\right\rangle f(x)\right\}+\frac{1}{2} \sum_{j, k} \frac{\partial^{2}}{\partial x_{j} \partial x_{k}}\left\{\left\langle H_{j}\left(t, x^{0}\right) H_{k}\left(t, x^{0}\right)\right\rangle f(x)\right\}+\ldots
$$


one can rewrite the Eq. (2.5) into the form

$$
W(x, t)=(1+L)\left\{\delta\left(x-x^{0}\right\} .\right.
$$

On the other hand, from Eqs (2.6), (2.7) it can be obtained another operator

$$
L^{\prime}=-\sum_{j} \frac{\partial}{\partial x_{j}}\left\langle H_{j}^{\prime}\left(t, x^{0}\right)\right\rangle+\frac{1}{2} \sum_{j, k} \frac{\partial^{2}}{\partial x_{j} \partial x_{k}}\left\langle H_{j}^{\prime}\left(t, x^{0}\right) H_{k}^{\prime}\left(t, x^{0}\right)\right\rangle+\ldots
$$

where $H_{j}^{\prime}(t, x)=\partial H_{j}(t, x) / \partial t$, operating analogically as the operator $L$ in Eq. (2.7) and from Eq. (2.8) one has

$$
\frac{\partial W(x, t)}{\partial t}=L^{\prime}\left\{\delta\left(x-x^{0}\right)\right\} .
$$

The Eqs. (2.8) and (2.9) yields the equation

$$
\frac{\partial W(x, t)}{\partial t}=L^{\prime}(1+L)^{-1}\{W(x, t)\} .
$$

Assuming that

$$
H_{j}(t, x)=\varepsilon H_{j 1}(t, x)+\varepsilon^{2} H_{j 2}(t, x)+\varepsilon^{3} H_{j 3}(t, x)+\varepsilon^{4} \ldots .
$$

the introduced above operators can be rewritten in the form

$$
\begin{aligned}
L= & -\varepsilon \sum_{j} \frac{\partial}{\partial x_{j}}\left\langle H_{j 1}\left(t, x^{0}\right)\right\rangle \\
& +\varepsilon^{2}\left\{-\sum_{j} \frac{\partial}{\partial x_{j}}\left\langle H_{j 2}\left(t, x^{0}\right)\right\rangle+\frac{1}{2} \sum_{j, k} \frac{\partial^{2}}{\partial x_{j} \partial x_{k}}\left\langle H_{j 1}\left(t, x^{0}\right) H_{k 1}\left(t, x^{0}\right)\right\rangle\right\}+\varepsilon^{3} \ldots \\
L^{\prime}= & -\varepsilon \sum_{j} \frac{\partial}{\partial x_{j}}\left\langle H_{j 1}^{\prime}\left(t, x^{0}\right)\right\rangle \\
& +\varepsilon^{2}\left\{-\sum_{j} \frac{\partial}{\partial x_{j}}\left\langle H_{j 2}^{\prime}\left(t, x^{0}\right)\right\rangle+\frac{1}{2} \sum_{j, k} \frac{\partial^{2}}{\partial x_{j} \partial x_{k}}\left\langle H_{j 1}^{\prime}\left(t, x^{0}\right) H_{k 1}^{\prime}\left(t, x^{0}\right)\right\rangle\right\}+\varepsilon^{3} \ldots \\
(1+L)^{-1}=1 & -L+\ldots=1+\varepsilon \sum_{j} \frac{\partial}{\partial x_{j}}\left\langle H_{j 1}\left(t, x^{0}\right)\right\rangle-\varepsilon^{2} \ldots
\end{aligned}
$$

Finally, taking into account the equality

$$
\begin{aligned}
& \frac{\partial}{\partial x_{j}}\left\langle H_{j 1}^{\prime}(t, x)\right\rangle \frac{\partial}{\partial x_{k}}\left\langle H_{k 1}(t, x)\right\rangle \\
& =\frac{\partial^{2}}{\partial x_{j} \partial x_{k}}\left\langle H_{j 1}^{\prime}(t, x)\right\rangle\left\langle H_{k 1}(t, x)\right\rangle-\frac{\partial}{\partial x_{j}}\left\langle\partial H_{j 1}^{\prime}(t, x) / \partial x_{k}\right\rangle\left\langle H_{k 1}(t, x)\right\rangle
\end{aligned}
$$

the equation $(2.10)$ can be rewritten as 


$$
\begin{aligned}
\frac{\partial W}{\partial t}= & -\varepsilon \sum_{j} \frac{\partial}{\partial x_{j}}\left[\left\langle H_{j 1}^{\prime}(t, x)\right\rangle W\right] \\
& -\varepsilon^{2} \sum_{j} \frac{\partial}{\partial x_{j}}\left[\left\langle H_{j 2}^{\prime}(t, x)\right\rangle W+\sum_{k} \frac{\partial\left\langle H_{j 1}^{\prime}(t, x)\right\rangle}{\partial x_{k}}\left\langle H_{k 1}(t, x)\right\rangle W\right] . \\
& +\varepsilon^{2} \sum_{j, k} \frac{\partial^{2}}{\partial x_{j} \partial x_{k}}\left[\left\langle H_{j 1}^{\prime}(t, x) H_{k 1}(t, x)\right\rangle W-\left\langle H_{j 1}^{\prime}(t, x)\right\rangle\left\langle H_{k 1}(t, x)\right\rangle W\right]+\varepsilon^{3} \ldots
\end{aligned}
$$

or in more compact form if ignoring the orders more than 2 of the parameter $\varepsilon$

$$
\frac{\partial W}{\partial t}+\sum_{j} \frac{\partial}{\partial x_{j}}\left[K_{j}(t, x) W\right]=\frac{1}{2} \sum_{j, k} \frac{\partial^{2}}{\partial x_{j} \partial x_{k}}\left[D_{j k}(t, x) W\right] .
$$

where

$$
\begin{aligned}
K_{j} & =\varepsilon\left\langle H_{j 1}^{\prime}+\varepsilon H_{j 2}^{\prime}\right\rangle+\varepsilon^{2} \sum_{k}\left\langle\frac{\partial H_{j 1}^{\prime}}{\partial x_{k}}\right\rangle\left\langle H_{k 1}\right\rangle ; \\
D_{j k} & =2 \varepsilon^{2}\left[\left\langle H_{j 1}^{\prime} H_{k 1}\right\rangle-\left\langle H_{j 1}^{\prime}\right\rangle\left\langle H_{k 1}\right\rangle\right] .
\end{aligned}
$$

The asymptotically approximate equation for PDF of arbitrary random process $X$ has the form that recalls the Fokker-Plank-Kolmogorov Equation for diffusional process.

\section{APPLICATION TO THE SYSTEMS OF STOCHASTIC INTEGRO-DIFFERENTIAL EQUATIONS}

Suppose that the process $X(t)$ is determined by the equation

$$
\begin{array}{r}
X_{j}^{\prime}(t)=\varepsilon^{2} A_{j}\left(X, t, \int_{-\infty}^{t} \varphi(t, s, X(s)) d s\right)+\varepsilon \sum_{k} B_{j k}\left(X, t, \int_{-\infty}^{t} \psi(t, s, X(s)) d s\right) \xi_{k}(t), \\
j=1, \ldots, n .
\end{array}
$$

where $\xi_{k}(t), k=1, \ldots, m$ are stationary random process with zero mean value and correlation functions

$$
R_{k l}(\tau)=\left\langle\xi_{k}(t) \xi_{l}(t+\tau)\right\rangle
$$

Letting

$$
X_{j}(t)=x_{j}^{0}+H_{j}\left(t, x^{0}\right)=x_{j}^{0}+\varepsilon H_{j 1}\left(t, x^{0}\right)+\varepsilon^{2} H_{j 2}\left(t, x^{0}\right)+\ldots,
$$

one will have

$$
X_{j}^{\prime}(t)=\varepsilon H_{j 1}^{\prime}\left(t, x^{0}\right)+\varepsilon^{2} H_{j 2}^{\prime}\left(t, x^{0}\right)+\ldots
$$

Using the Eqs. (3.3) and (3.4), one can calculate the integrals

$$
\begin{aligned}
\int_{-\infty}^{t} \varphi(t, s, X(s)) d s & =\int_{-\infty}^{t} \varphi\left(t, s, x^{0}\right) d s+\varepsilon \sum_{j} \int_{-\infty}^{t} \frac{\partial \varphi\left(t, s, x^{0}\right)}{\partial x_{j}} H_{j 1}\left(s, x^{0}\right) d s+\varepsilon^{2} \ldots \\
& =\varphi^{0}\left(t, x^{0}\right)+\varepsilon \varphi^{1}\left(t, x^{0}\right)+\ldots
\end{aligned}
$$




$$
\begin{aligned}
\int_{-\infty}^{t} \psi(t, s, X(s)) d s & =\int_{-\infty}^{t} \psi\left(t, s, x^{0}\right) d s+\varepsilon \sum_{j} \int_{-\infty}^{t} \frac{\partial \psi\left(t, s, x^{0}\right)}{\partial x_{j}} H_{j 1}\left(s, x^{0}\right) d s+\varepsilon^{2} \ldots \\
& =\psi^{0}\left(t, x^{0}\right)+\varepsilon \psi^{1}\left(t, x^{0}\right)+\ldots
\end{aligned}
$$

Further one can get

$$
\begin{aligned}
& A_{j}\left(X, t, \int_{-\infty}^{t} \varphi(t, s, X(s)) d s\right)= A_{j}^{0}\left(x^{0}, t\right)+\varepsilon \sum_{k} \frac{\partial A_{j}^{0}}{\partial x_{k}} H_{k 1} \\
&-\varepsilon \sum_{k l} \int \frac{\partial A_{j}^{0}}{\partial \varphi_{k}^{0}} \frac{\partial \varphi_{j}^{0}\left(s, x^{0}\right)}{\partial x_{l}} H_{l 1}^{\prime}\left(s, x^{0}\right) d s+\ldots \\
& B_{j k}\left(X, t, \int_{-\infty}^{t} \psi(t, s, X(s)) d s\right)= B_{j k}^{0}\left(x^{0}, t\right)+\varepsilon \sum_{m} \frac{\partial B_{j k}^{0}}{\partial x_{m}} H_{m 1} \\
&-\varepsilon \sum_{m l} \int \frac{\partial B_{j k}^{0}}{\partial \psi_{m}^{0}} \frac{\partial \psi_{m}^{0}\left(s, x^{0}\right)}{\partial x_{l}} H_{l 1}^{\prime}\left(s, x^{0}\right) d s+\ldots \\
& A_{j}^{0}(t, x)=A_{j}\left(t, x, \varphi^{0}(t, x)\right), B_{j}^{0}(t, x)=B_{j}\left(t, x, \psi^{0}(t, x)\right) .
\end{aligned}
$$

Substituting Eqs (3.4) and (3.5) into Eq. (3.1) follows the equations

$$
\left.\begin{array}{c}
\left\langle H_{j 1}^{\prime}\left(t, x^{0}\right\rangle\right)=\sum_{k} B_{j k}^{0}\left(t, x^{0}\right)\left\langle\xi_{k}(t)\right\rangle=0= \\
\left\langle H_{j 1}\left(t, x^{0}\right)\right\rangle=\int_{-\infty}^{t} \sum_{k} B_{j k}^{0}\left(s, x^{0}\right)\left\langle\xi_{k}(s)\right\rangle d s \\
\left\langle H_{j 2}^{\prime}(t, x)\right\rangle=A_{j}^{0}(t, x) \\
+\sum_{k, m}\left\{\frac{\partial B_{J K}^{0}}{\partial x_{m}} \int_{-\infty}^{t} \sum_{l} \frac{\partial B_{J K}^{0}}{\partial \psi_{m}^{0}} \int_{-\infty}^{t} \sum_{l, h} \frac{\partial \psi_{m}^{0}}{\partial x_{l}} B_{l h}^{0}(s, x) R_{k h}(s) d s\right.
\end{array}\right\} .
$$

Using the obtained expressions, one can calculate the coefficients (2.12) for Eq. (2.11)

$$
K_{j}(t, x)=\varepsilon^{2} A_{J}^{0}(t, x)+\varepsilon^{2} \sum_{k, m}\left\{\begin{array}{l}
\frac{\partial B_{j k}^{0}}{\partial x_{m}} \int_{0}^{\infty} \sum_{l} B_{m l}^{0}(t-s, x) R_{k l}(s) d s \\
-\frac{\partial B_{j k}^{0}}{\partial \psi_{m}^{0}} \int_{0}^{\infty} \sum_{l, h} \frac{\partial \psi_{m}^{0}}{\partial x_{l}} B_{l h}^{0}(t-s, x) R_{k h}(s) d s
\end{array}\right\} ;
$$




$$
D_{i j}(t, x)=2 \varepsilon^{2} \sum_{k, m} B_{i k}^{0}(t, x) \int_{0}^{\infty} B_{j m}^{0}(t-s, x) R_{k m}(s) d s .
$$

In particularity, functions $\varphi=\psi=0$, i.e. equations (3.1) are differential, coefficients (3.8) may be simplified as

$$
\begin{gathered}
K_{j}(t, x)=\varepsilon^{2} A_{J}^{0}(t, x)+\varepsilon^{2} \sum_{k, m} \frac{\partial B_{j k}^{0}}{\partial x_{m}} \int_{0}^{\infty} \sum_{l} B_{m l}^{0}(t-s, x) R_{k l}(s) d s \\
D_{i j}(t, x)=2 \varepsilon^{2} \sum_{k, m} B_{i k}^{0}(t, x) \int_{0}^{\infty} B_{j m}^{0}(t-s, x) R_{k m}(s) d s
\end{gathered}
$$

Moreover, if the process $\xi_{k}(t), k=1, \ldots, m$ is white noises, i. e.

$$
R_{k l}(\tau)=\left\langle\xi_{k}(t) \xi_{l}(t+\tau)\right\rangle=\sigma_{j} \gamma_{k j} \delta(\tau)
$$

with the notation $\gamma_{j k}=1$ if $k=j$ and $=0$ for $j \neq k$, the coefficients (2.9) become

$$
\begin{aligned}
& K_{j}(t, x)=\varepsilon^{2} A_{J}^{0}(t, x)+\varepsilon^{2} \sum_{k, m} \sigma_{k} \frac{\partial B_{j k}^{0}}{\partial x_{m}} B_{m k}^{0}(t, x) \\
& D_{i j}(t, x)=2 \varepsilon^{2} \sum_{k} B_{i k}^{0}(t, x) B_{j k}^{0}(t, x) .
\end{aligned}
$$

Equation (2.11) with coefficients (3.10) were obtained firstly by Stratonovich [2].

\section{APPLICATION TO SYSTEMS WITH DELAY}

It's not difficult to verify that the system of differential equations with delay

$$
\begin{aligned}
X_{j}^{\prime}(t)= & \varepsilon^{2} A_{j}(X, t, \alpha(t-\Delta, X(t-\Delta))) \\
& +\varepsilon \sum_{k} B_{j k}(X, t, \beta(t-\Delta, X(t-\Delta))) \xi_{k}(t), j=1, \ldots, n .
\end{aligned}
$$

is a particularity of the system of equations (3.1) with

$$
\begin{aligned}
& \varphi(t, X, s, X(s))=\alpha(s, X(s)) \delta(t-\Delta-s) \\
& \psi(t, X, s, X(s))=\beta(s, X(s)) \delta(t-\Delta-s) .
\end{aligned}
$$

So that

$$
\varphi^{0}(t, x)=\alpha(t-\Delta, x) ; \quad \psi^{0}(t, x)=\beta(t-\Delta, x)
$$

and

$$
A_{j}^{0}(t, x)=A_{j}(x, t, \alpha(t-\Delta, x)), \quad B_{j}^{0}(t, x)=B_{j}(x, t, \beta(t-\Delta, x)) .
$$


In that case coefficients of the equation (2.11) can be determined as

$$
\begin{aligned}
& K_{j}(t, x)=\varepsilon^{2} A_{J}^{0}(t, x)+\varepsilon^{2} \sum_{k, m}\left\{\begin{array}{l}
\frac{\partial B_{j k}^{0}}{\partial x_{m}} \int_{0}^{\infty} \sum_{l} B_{m l}^{0}(t-s, x) R_{k l}(s) d s \\
-\frac{\partial B_{j k}^{0}}{\partial \beta_{m}} \int_{0}^{\infty} \sum_{l, h} \frac{\partial \beta_{m}}{\partial x_{l}} B_{l h}^{0}(t-s, x) R_{k h}(s) d s
\end{array}\right\} ; \\
& D_{i j}(t, x)=2 \varepsilon^{2} \sum_{k, m} B_{i k}^{0}(t, x) \int_{0}^{\infty} B_{j m}^{0}(t-s, x) R_{k m}(s) d s .
\end{aligned}
$$

If the process $\xi_{k}(t), k=1, \ldots, m$ is white noise, the last equations become

$$
\begin{gathered}
K_{j}(t, x)=\varepsilon^{2} A_{J}^{0}(t, x)+\varepsilon^{2} \sum_{k, m} \sigma_{k}\left[\frac{\partial B_{j k}^{0}}{\partial x_{m}} B_{m k}^{0}(t, x)-\frac{\partial B_{j k}^{0}}{\partial \beta_{m}} \sum_{l} \frac{\partial \beta_{m}}{\partial x_{l}} B_{l k}^{0}(t, x)\right] ; \\
D_{i j}(t, x)=2 \varepsilon^{2} \sum_{k} \sigma_{k} B_{i k}^{0}(t, x) B_{j k}^{0}(t, x) .
\end{gathered}
$$

Let's consider the equation

$$
u^{\prime \prime}(t)+\omega^{2} u(t)=\mu f\left(t, u, u^{\prime}, u(t-\Delta), u^{\prime}(t-\Delta)\right)+\sqrt{\mu} \sum_{k} g_{k}\left(t, u, u^{\prime}\right) \xi_{k}(t) .
$$

Using the variable transform

$$
u(t)=X_{c}(t) \cos \omega t+X_{s}(t) \sin \omega t ; u^{\prime}(t)=\omega\left[X_{s}(t) \cos \omega t-X_{c}(t) \sin \omega t\right],
$$

the Eq. (4.7) may be transformed into the system of the form (4.1) with $\mu=\varepsilon^{2}$ and

$\alpha_{1}(t, x)=x_{c}(t) \cos \omega t+x_{s}(t) \sin \omega t ; \alpha_{2}(t, x)=\omega\left[x_{s}(t) \cos \omega t-x_{c}(t) \sin \omega t ; \beta_{1}=\beta_{2}=0\right]$.

So that

$$
\begin{aligned}
& \alpha_{1}(t-\Delta, x)=x_{c} \cos \omega(t-\Delta)+x_{s} \sin \omega(t-\Delta) ; \\
& \alpha_{2}(t-\Delta, x)=\omega\left[x_{s} \cos \omega(t-\Delta)-x_{c} \sin \omega(t-\Delta)\right] .
\end{aligned}
$$

$A_{1}^{0}=\frac{1}{\omega_{1}} f[t, x] \cos \omega t ; \quad A_{2}^{0}=-\frac{1}{\omega} f[t, x] \sin \omega t ; \quad B_{11}^{0}=\frac{1}{\omega} g_{1}[t, x] \cos \omega t ;$

$$
B_{12}^{0}=\frac{1}{\omega} g_{2}[t, x] \cos \omega t ; \quad B_{21}^{0}=\frac{1}{\omega} g_{1}[t, x] \sin \omega t ; \quad B_{22}^{0}=-\frac{1}{\omega} g_{2}[t, x] \sin \omega t ;
$$

$\left.f[t, x]=f\left[t, x_{c} \cos \omega t+x_{s} \sin \omega t, \omega\left[x_{s} \cos \omega t-x_{c} \sin \omega t\right], \alpha_{1}(t-\Delta, x)\right), \alpha_{2}(t-\Delta, x)\right] ;$ $g_{1,2}[t, x]=g_{1,2}\left[t, x_{c} \cos \omega t+x_{s} \sin \omega t, \omega\left[x_{s} \cos \omega t-x_{c} \sin \omega t\right]\right]$.

Equations (4.5) would be used for determining the coefficients (4.5) of the equation 


\section{CASE STUDIES}

\subsection{Elementary example}

Consider the equation

$$
X^{\prime}(t)=-\mu \int_{-\infty}^{t} e^{-\lambda(t-s)} X(s) d s+\sqrt{\mu} \sigma \xi(t),
$$

with white noise $\xi(t)$. Applying the developed above theory, one will have the equation for PDF

$$
\begin{aligned}
& \frac{\partial W(x, t)}{\partial t}+\frac{\partial}{\partial x}[K W]=\frac{1}{2} \frac{\partial^{2}}{\partial x^{2}}[D W], \\
& K(t, x)=-\frac{\mu x}{\lambda}, \quad D(t, x)=\mu \sigma^{2},
\end{aligned}
$$

that yields the stationary solution

$$
W_{0}(x)=[\sigma \sqrt{\lambda \pi}]^{-1} \exp \left\{-x^{2} / \lambda \sigma^{2}\right\} .
$$

The solution, as mentioned above, is approximate with respect to the small parameter $\mu$ (even it does not depend on the parameter). To see how the solution is accurate, let's consider equation (5.1) from other point of view. Assuming

$$
Y(t)=\int_{-\infty}^{t} e^{-\lambda(t-s)} X(s) d s
$$

leads to $Y^{\prime}(t)=X(t)-\lambda Y(t)$ and at the end one gets the system

$$
X^{\prime}=-\mu Y+\sqrt{\mu} \sigma \xi(t) ; \quad Y^{\prime}=-\lambda Y+X .
$$

Well-known FPK equation for the system (5.4) results immediately in stationary solution

$$
W_{0}(x, y)=C \cdot \exp \left\{-\frac{\lambda}{\mu \sigma^{2}}\left[x^{2}-2 \lambda x y+\left(\lambda^{2}+\mu\right) y^{2}\right]\right\},
$$

that allows to get the stationary solution for $x$ as

$$
W_{0}^{E}(x, \mu)=C_{X} \exp \left[-\frac{x^{2}}{\lambda \sigma^{2}\left(1+\frac{\mu}{\lambda^{2}}\right)}\right] .
$$

From the condition $\int_{-\infty}^{+\infty} W_{0}(x) d x=1$ one gets $C_{X}=\left[\frac{\lambda}{\pi \sigma^{2}\left(\lambda^{2}+\mu\right)}\right]^{1 / 2}$, so that

$$
W_{0}^{E}(x, \mu)=\left[\sigma \sqrt{\pi \lambda\left(1+\frac{\mu}{\lambda^{2}}\right)}\right]^{-1} \exp \left[-\frac{x^{2}}{\lambda \sigma^{2}\left(1+\frac{\mu}{\lambda^{2}}\right)}\right] .
$$

It's easily to see that

$$
W_{0}(x)=\lim _{\mu \rightarrow 0} W_{0}^{E}(x, \mu)
$$




\subsection{The Van de Pol's system with delay}

Now we investigate the system

$$
u^{\prime \prime}(t)+\omega^{2} u(t)=\mu \alpha\left[1-u^{2}(t-\Delta)\right] u^{\prime}(t-\Delta)-\mu h u^{\prime}(t)+\sqrt{\mu} \sigma \xi(t) .
$$

Applying the formulas (4.5)-(4.8) allows us to get the stationary solution of the equation (2.11) in the form

$$
W_{0}\left(x_{c}, x_{s}\right)=C \cdot \exp \left\{-\frac{\omega^{2}}{\sigma^{2}}\left(x_{c}^{2}+x_{s}^{2}\right)\left[h-\alpha\left(1-\frac{x_{c}^{2}+x_{s}^{2}}{8}\right) \cos \omega \Delta\right]\right\}
$$

or the PDF in variables of amplitude and phase will take the form

$$
W_{0}(a)=C \cdot a \cdot \exp \left\{-\frac{\omega^{2} a^{2}}{\sigma^{2}}\left[h-\alpha\left(1-\frac{a^{2}}{8}\right) \cos \omega \Delta\right]\right\} .
$$

The solution (5.9) gives an equation for amplitude of stationary vibration of maximal probability as follows

$$
(\alpha \cos \omega \Delta) a^{4}-4(h-\alpha \cos \omega \Delta) a^{2}+2 \frac{\sigma^{2}}{\omega^{2}}=0 .
$$

The equation shows that if $\sigma=0$, i.e. there is no random excitation, the system cannot be excited under the condition $h-\alpha \cos \beta \omega \Delta \geq 0$. Otherwise, the system is self-excited with the amplitude of vibration $a_{0}=2 \sqrt{1-h(\alpha \cos \omega \Delta)^{-1}}$. This result were obtained by Rubanik [7] in 1969.

In the case of random excitation, the system always is excited with vibration amplitude monotony increasing with parameter $\rho=h-\alpha \cos \omega \Delta$, if $\sigma^{2}>8 h \omega^{2}$ and monotony decreasing with the parameter $\rho$, if $\sigma^{2}<8 h \omega^{2}$. In the case, if $\sigma^{2}=8 h \omega^{2}$, the vibration amplitude equals permanently to 2, exactly as in the classical Van de Pol's system, regardless of delay.

\section{CONCLUSION}

In this paper the following results have been presented:

- An equation for probability density function of arbitrary stochastic process has been constructed based on its asymptotic expansion that recalls the Fokker-PlankKolmogorov equation for diffusion process. The developed equation is approximate only but it can be used for study numerous weakly nonlinear oscillation systems.

- The theory has been applied to processes given by stochastic integro-differential equations or differential equation with delay. This is a further developments of the FPKE method to study non-diffusion processes.

- Illustrating examples have validated the applicability and effectiveness of the developed approach.

Acknowledgement. The author is thankful very much to Editor in Chief of Vietnam Journal of Mechanics for inviting his to contribute the paper and also to Vietnam Science Fund for financial supports for completing this work. 


\section{REFERENCES}

1. A. A. Andronov, L. S. Pontriaghin and A. Vitt, On a statistical investigation of dynamical systems, Journal of Experimental and Technical Physics 3 (3) (1933) 165-180.

2. R. L. Stratonovich, Selected Problems in the Theory of Fluctuations in Radio-Technique, Moscow, 1961, 558p.

3. R. Z. Khasminskii, On stochastic processes determined by differential equation witj small parameter, Journal of Theory of Probability and Its Application 11 (2) (1966) 240-259.

4. Nguyen Van Dao, Nonlinear Oscillations of High Order Systems, NCSR of Vietnam, 1979. 64p.

5. Nguyen Tien Khiem, On the probability density function of the processes given by integrodifferential equation, Ukrainian Mathematical Journal 35 (2) (1983) 227-234.

6. H. J. Kushner, Approximation and Weak Convergence Method for Random Processes with Application to Stochastic Systems Theory, Cambridge, MA, MIT Press, 1984, 269p

7. V. P. Rubanik, Oscillations in Complex Quasy-Linear System with Delay, Minsk, 1985, 143p.

8. Nguyen Tien Khiem, Application of the Fokker-Plank-Kolmogorov Equation Method to study random vibration of systems with hysteresis, Doctor of Science Dissertation, Kiev, 1991, 155p.

Received June 19, 2007.

\section{MộT CÁCH TIẾP CẬN NGHIÊN CỨU DAO DộNG CÁC HỆ NGẪU NHIÊN BẰNG PHƯƠNG PHÁP TIỆM CẬN}

Trong báo cáo này trình bày việc thiết lập phương trình Fokker-Plank-Kolmogorov cho các quá trình không phải là quá trình Markov. Sau đó áp dụng cho các quá trình được xác định bằng phương trình vi tích phân ngẫu nhiên và vi phân ngẫu nhiên có chậm. Các phương trình nhận được được minh họa trên các ví dụ cụ thể để chứng minh tính đúng đắn và khả năng ứng dụng của phương pháp. 